\title{
Lichenoid Contact Dermatitis Induced by Semen Sinapis Albae
}

Guo $\mathrm{S}^{1,2}$, Jiang J-F², Tan $\mathrm{C}^{2}$

${ }^{1}$ First Clinical College, Nanjing University of Chinese Medicine, Nanjing, China

${ }^{2}$ Department of Dermatology, Affiliated Hospital of Nanjing University of Chinese Medicine, Nanjing, China.

J Investig Allergol Clin Immunol 2018; Vol. 28(5): 350-352 doi: 10.18176/jiaci.0286

Key words: Contact Dermatitis. Lichenoid. Acupoint. Semen Sinapis Albae.

Palabras clave: Dermatitis de contacto. Liquenoide. Acupuntos. Semillas de mostaza blanca.

The clinical manifestations of contact dermatitis are varied and present as either eczematous or noneczematous eruptions [1]. Lichenoid contact dermatitis (LCD) is a rare noneczematous form of contact dermatitis. It is caused by multiple factors, including exposure to plants, drugs, and chemicals [2]. Nonetheless, to the best of our knowledge, lichenoid contact dermatitis induced by an acupoint herbal patch (AHP) with Semen Sinapis Albae (SSA) has never been reported.

A 55-year-old Chinese man was evaluated for a 4-month history of nummular eruptions on an area treated with AHP. He had a 14-year history of chronic obstructive pulmonary disease with repeated exacerbations in winter. Following a prescription from a herbalist, AHP with SSA seeds was applied twice, on June 12 and 22, 2017. Twenty-four hours after the second application, each patch was detached with no noticeable cutaneous abnormalities. One month later, pruritic erythema and pin-sized papules appeared at the site. The primary eruptions became progressively pigmented and coalesced and did not fade with discontinuation of SSA or recede with the administration of topical corticosteroids and antihistamines. The patient was otherwise in good health and denied exposure to SSA before treatment of AHP. Skin examination revealed multiple nummular lesions distributed bilaterally along the spine, each overlaying a specific Back-shu acupoint. The lesions comprised demarcated erythematous macules and plaques. Some of the lesions were entirely pigmented or overlapped with pin-sized papules (Figure, A). A skin biopsy displayed parakeratosis, acanthosis, and focal spongiosis in the epidermis. An infiltration of band-like mononuclear cells was visible along the dermoepidermal junction. There was no evidence of liquefaction of the basal cell layer or hyaline bodies (Figure, B). The patient was diagnosed with lichenoid contact dermatitis induced by SSA. The lesions did not improve with 1 month of topical application of $0.1 \%$ tacrolimus and persisted during follow-up.

The remedy of AHP was firstly introduced in the Chinese medical book Prescriptions for Fifty-two Diseases written in 


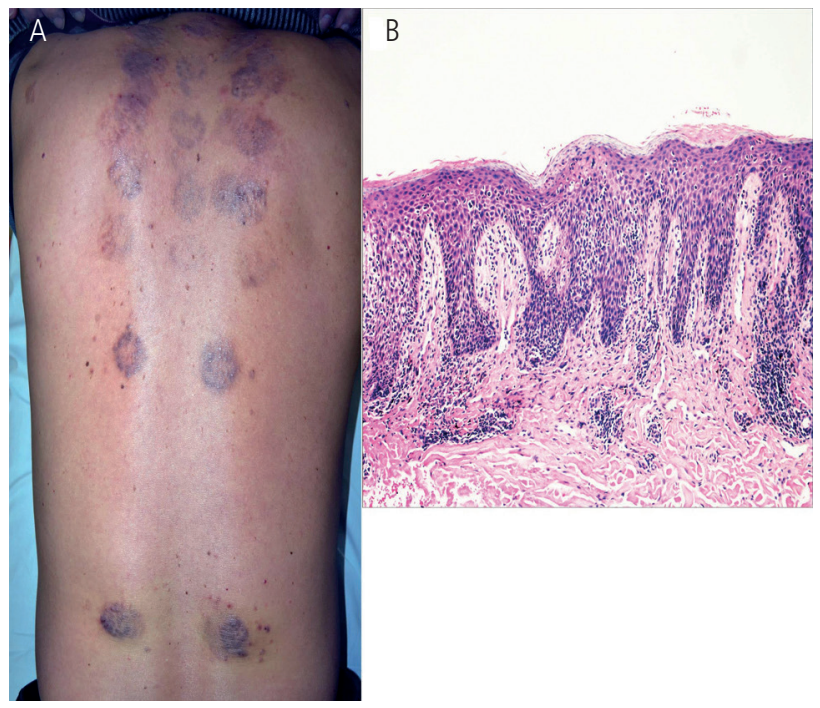

Figure. A, Lesions on the patient's back presenting as discoid demarcated erythematous macules and plaques. These eruptions are distributed bilaterally along the spine, and each overlays a specific Back-shu acupoint. B, Parakeratosis, acanthosis, and focal spongiosis in the epidermis. An infiltration of band-like mononuclear cells can be seen along the dermoepidermal junction. Liquefaction of the basal cell layer and hyaline bodies were not observed (hematoxylin-eosin, $\times 10$ ).

the Western Han Dynasty (206 BC to 25AD). This traditional approach involves applying Chinese herbs onto specific acupoints. The most common herbs applied during the AHP are Semen Sinapis Albae (白芥子), Herba Asari (细辛), Radix Euphorbiae kansui (甘遂), and Rhizoma Corydalis (延胡索) [3], which can be used individually or as a combination of several herbs. AHP has already become a prevalent treatment option for variable chronic diseases. Millions of patients in China undergo such complimentary therapy annually on the 3 dog days because of the highest potential of the Yang at these times. In traditional Chinese medicine, it is believed that AHP reverses Yang deficiency syndrome [4]. Therefore, AHP is carried out during the dog days to ensure early intervention in a disease that is quiescent in summer with a high possibility of relapse or exacerbation in winter. Chronic obstructive pulmonary disease, asthma, and chronic bronchitis all fall within the spectrum of diseases suitable for AHP $[3,4]$.

SSA takes the form of small pungent seeds of the Brassicaceae family. It is widely used not only as a seasoning and spice, but also as a medicinal plant $[5,6]$. Since SSA disperses sputum and eliminates cold to invigorate the Yang, AHP may function synergistically with the herb SSA if applied on the 3 summer dog days. Acute allergic reactions have been reported during topical application of AHP with SSA. The most common involve eczematous lesions with an itching/burning sensation shortly after exposure [5]. However, noneczematous presentations, such as lichenoid contact dermatitis, are quite rare.

Lichenoid contact dermatitis is a particularly unusual type of contact dermatitis caused by exposure to amalgams and nickel [7]. Other uncommon causative allergens include methylisothiazolinone, aminoglycoside antibiotics, methacrylic acid esters, methylchloroisothiazolinone, paraphenylenediamine, and epoxy resins. Histopathology reveals hyperkeratosis, acanthosis, mild spongiosis, and exocytosis. An infiltration of band-like mononuclear cells is noted along the dermoepidermal junction, although there is no evidence of liquefaction of the basal cell layer or hyaline bodies [1,2]. Lichenoid contact dermatitis should be differentiated from other types of noneczematous contact dermatitis such as erythema multiforme, purpura, and the lymphomatoid, pigmented, pustular, dyshidrosiform, and lichenoid subtypes [8]. The clinical and histopathological data recorded in the present case made these diagnoses unlikely.

We propose that SSA may act as a major contact allergen, so that a single exposure could sensitize the predisposing individual even 1 month later. Similar reactions have been observed with epoxy resin, acrylates, some hair dyes, and black henna for tattoos [9]. However, the pathogenesis of lichenoid contact dermatitis with SSA remains to be elucidated. We present the first case of lichenoid contact dermatitis after exposure to SSA. Clinicians should include lichenoid contact dermatitis among the differential diagnoses when there is microscopic evidence of interface dermatitis.

\section{Funding}

The study was funding in part by the National Natural Science Foundation of China (Grant No. 81173400).

\section{Conflicts of Interest}

The authors declare that they have no conflicts of interest.

\section{References}

1. Kränke B, Schuster C. Contact dermatitis: relevant differential diagnoses, simulators, and variants. J Dtsch Dermatol Ges. 2015;13:1073-88.

2. Bonamonte $D$, Foti $C$, Vestita $M$, Angelini G. Noneczematous contact dermatitis. ISRN Allergy. 2013;2013:361746.

3. Liu H. Acupuncture Combined with Acupoint Application Improves Symptoms, Daily Life Quality and Lung Function in Chronic Obstructive Pulmonary Disease Patients during Acute Exacerbation. Zhen Ci Yan Jiu. 2016:41:251-4.

4. Du LN, ZhuWN, Liu XY, Chen W Y, Yu X, Li M, et al. Preparation, in vitro and in vivo evaluation of cataplasm of white mustard seed varnish to prevent asthma. Zhongguo Zhong Yao Za Zhi. 2014;39:4596-602.

5. Wen $B L$, Liu BY, Jin P, Wang XF, Xiang XX, Liu XF, et al. Clinical research of acupoint application for "treatment of winter disease in summer" used to prevent and treat bronchial asthma in children. J Tradit Chin Med.2012;32:31-9.

6. Li J, Jin HZ. Allergic contact dermatitis caused by Chinese herbal medicine, white mustard seed. J Dermatol. 2013;40:6970.

7. Thanyavuthi A, Boonchai W, Kasemsarn P. Amalgam contact allergy in oral lichenoid lesions.Dermatitis. 2016;27:21521. 
8. Schoel J, Tilgen W, Frosch PJ. Lichenoid contact eczema caused by color film developer. Hautarzt.1991;42:251-3.

9. Raymond J, Konya J, Bakis-Petsoglou S. Lichenoid contact dermatitis secondary to methylisothiazolinone (MI). JAAD Case Rep.2016;2:380-3.

Manuscript received March 20, 2018; accepted for publication June 18, 2018.

Cheng Tan Department of Dermatology Jiangsu Provincial Hospital of Traditional Chinese Medicine 155 Hanzhong Road, Nanjing, 210029 E-mail: tancheng@yeah.net 